\section{Segmental Box Girder: Effect of Spatial RaNdom Variability OF Material ON DEFLECTIONS}

By Zdeněk P. Bažant,' Fellow, ASCE, and Joong-Koo Kim²

\section{INTRODUCTION}

In a preceding paper (Bažant and Kim 1989), a method of probabilistic prediction of the confidence limits on long-time deflections and internal forces of prestressed concrete segmental box-girder bridges was developed. The sampling method was used and predictions were improved by Bayesian updating on the basis of short-time measurements. The entire analysis, including its Bayesian aspect, was made according to the latin hypercube sampling scheme, which represents an efficient way to solve the problem. The analysis considered the uncertainties of shrinkage, basic creep, drying creep, environmental relative humidity, concrete strength, water-cement ratio, gravelcement ratio and cement content-altogether eight random material parameters of given distributions, assumed to be normal.

One aspect of statistical variability, however, was neglected in the preceding work. It was assumed that although the material compliance function has a random multiplicative factor, the value of this factor is constant throughout the entire structure. This is a simplification. In reality, the multiplicative factor on the compliance exhibits a spatial variability; it is a random field in space.

The purpose of this brief note is to extend the preceding formulation to include, in a simplified manner, the effect of spatial random variability of the compliance multiplicative factor. Development of the basic theory is not repeated from the preceding paper (Bažant and Kim 1989).

\section{Mathematical Formulation}

The compliance function of concrete, which represents the strain at age caused by unit stress applied at age $t^{\prime}$, may be written in the form

$J\left(t, t^{\prime}\right)=J_{0}\left(t, t^{\prime}\right) \frac{1}{1+\alpha}$

in which $J_{0}\left(t, t^{\prime}\right)=$ material compliance function without any spatial variability, the same as given in the preceding paper (Bažant and Kim 1989); and $\alpha=$ a random variable of zero mean, which is introduced here to describe the random spatial variability. The random field $\alpha$ can be character-

${ }^{\text {I Walter P. Murphy Prof. of Civ. Engrg., McCormick School of Engrg. and Appl. }}$ Sci.. Northwestern Univ., Evanston, IL 60208-3109.

'Grad. Res. Asst., McCormick School of Engrg. and Appl. Sci., Northwestern Univ., Evanston, IL.

Note. Discussion open until January 1, 1992. To extend the closing date one month, a written request must be filed with the ASCE Manager of Journals. The manuscript for this paper was submitted for review and possible publication on February 28, 1990. This paper is part of the Journal of Structural Engineering, Vol. 117, No.
for 8. August, 1991. OASCE, ISSN 0733-9445/91/0008-2542/\$1.00+\$.15 per page. Paper No. 26097 ized by the spatial autocorrelation function or, equivalently, by discretizing its Fourier transform. Thus, the random field can be approximated as a sum of $n$ sinusoidal components

$\alpha=\sum_{i=1}^{n} A_{i} f_{i}(x, y)$

$f_{i}(x, y)=\cos \left(\frac{2 \pi x}{L_{i x}}+\psi_{i j}\right) \cos \left(\frac{2 \pi y}{L_{i y}}+\phi_{i j}\right)$

in which the amplitudes $A_{i}=$ random variables of zero mean; the phase angles $\psi_{i j}$ and $\phi_{i j}=$ random variables, which have a uniform distribution over the interval $(0,2 \pi) ; x, y=$ horizontal and vertical coordinates of the points in the box girder of a prestressed concrete bridge [see Bažant and $\operatorname{Kim}(1989)$ ]; $L_{i x}$ and $L_{i y}=$ wavelengths of the periodic components of the random field in the horizontal and vertical directions; and subscript $j$ refers to the $j$ th segment of the segmentally cast box girder. In practical calculations, we will consider, for lack of deeper knowledge, only two terms in (2) $(i=1,2)$.

Eq. (2) describes in the most simple manner the fact that if at a certain point of the girder the random realization is $\alpha=a$, then at points not too far away [say $1 \mathrm{ft}(30.5 \mathrm{~cm})$ away] the random realization of $\alpha$ is likely to be close to $a$, and at points farther away it is likely to be quite different This is the basic aspect of spatial correlation. It would be meaningless to assume that the random values of $\alpha$ at very closely spaced points are independent random variables. In fact, it can be shown that if the values at each two points, even if infinitely closely spaced, were uncorrelated, then the structure would behave exactly in the same way as a structure in which $\alpha=0$ everywhere. Thus, spatial correlation must inevitably be assumed to obtain meaningful results.

It must be recognized that if there are only a limited number of sinusoida components, then there exists a certain length $L$ of periodicity of the function in (2). At points whose distance is $L$, the values of $\alpha$ are therefore equal, i.e., perfectly correlated. This aspect of (2) is of course unrealistic. It can be avoided only by taking infinitely many components. However, the error due to this correlation is negligible if the length $L$ of periodicity is sufficiently large. In our subsequent calculations we assume that the length of periodicity is sufficiently larger than the length of the bridge segments, as well as the depth of the layer of the box girder. Then, the aforementioned long-range periodicity is practically irrelevant, since the fields of $\alpha$ in dif ferent segments are assumed to be entirely uncorrelated.

The reason for spatial correlation lies in the method of production and casting of concrete. Obviously there will be a high correlation among the material parameters of the elements of concrete coming from the same batch of concrete. The entire batch of concrete is normally cast into one place in the girder. Thus, adjacent points are likely to come from the same batch and thus be strongly correlated. On the other hand, points farther away are likely to come from a different batch and thus be weakly correlated.

Aside from this aspect, correlation is also set by environmental conditions at the time of curing and casting. Closely spaced points in the material are likely to be cast at the same time and thus exposed to the same humidity 
and temperature history. On the other hand, points located farther apart are likely to have been, cast at different times and thus exposed to different histories. Furthermore, there is unlikely to be much correlation between the materials properties in different bridge segments. Inevitably, they would be cast from different batches of concrete and thus at very different times.

Also, there is likely to be a greater correlation in the horizontal direction than the vertical direction, because concrete is cast in horizontal layers that are long and thin. For this reason the value of $L_{i x}$ should be larger than that of $L_{i}$.

Representing the random variability in the form of (2) lends itself easily to the random sampling approach. In the preceding work (Bažant and Kim 1989) there were eight random parameters $\theta_{1}, \ldots, \theta_{8}$, as already mentioned (none of them depend on $x$ and $y$ ). The parameters $A_{i}, \psi_{i j}$, and $\phi_{i j}$ must now be included as additional random parameters $\theta_{9}, \theta_{10} \ldots$

According to the latin hypercube sampling scheme as described by Bažant and Kim (1989), the cumulative distributions of the additional random parameters are divided into horizontal layers of equal probability. Projections of the centroids of these layers yield sampling values of these parameters. As described in the previous work, the calculation of the statistics of the response is reduced to a number of deterministic runs for various randomly sampled values of parameters $\theta_{k}$. Previously only eight random parameters were sampled; now the latin hypercube sampling scheme is generalized by also including randomly sampled values of the additional parameters $A_{i}$, $\psi_{i j}$ and $\phi_{i j}$ [for details see Bažant and Kim (1989)]. The choice of their values is explained following (5).

\section{Numerical Calculations of Deflection Variability}

Proceeding in the same way as in the previous work, we consider two cases: (1) $n=1$ [i.e., only one term in the sum in (2)], with $A_{1}=0.2$; and (2) $n=2$ [i.e., two terms in the sum in (2)], with $A_{1}$ and $A_{2}$ chosen to be equal and $A_{1}=A_{2}=\sqrt{0.02}=0.1412$. Disregarding the effect of the boundaries, the variance of $\alpha$ is calculated as

$\operatorname{Var}(\alpha)=\lim _{L \rightarrow \infty}\left\{\frac{1}{4 L^{2}} \int_{-L}^{L} \int_{-L}^{L}\left[A_{l} f_{i}(x, y)\right]^{2} d x d y\right\}$

Substituting (2) into (3), we get

for $n=1: \quad \operatorname{Var}(\alpha)=\frac{A_{1}^{2}}{4}=0.01$

for $n=2: \quad \operatorname{Var}(\alpha)=\operatorname{Var}(\alpha)_{i=1}+\operatorname{Var}(\alpha)_{i=2}=\frac{A_{1}^{2}+A_{2}^{2}}{4}=0.01$

The values of $A_{i}$ are chosen so as to give the same variance of $\alpha$ in both examples. Since $\operatorname{Var}(\alpha)=\omega^{2}$, the present choice corresponds to coefficient of variation $\omega=0.1$. This value of $\omega$ is chosen to be the same as the coefficient of variation of the elastic modulus measured at the construction site, as reported by Shiu et al. (1983). In absence of other measurements, this is probably the best estimate of $\omega$ one can make. The values of $L_{i x}$ and $L_{i y}$, which may be interpreted as the maximum distances of correlation, are cho-
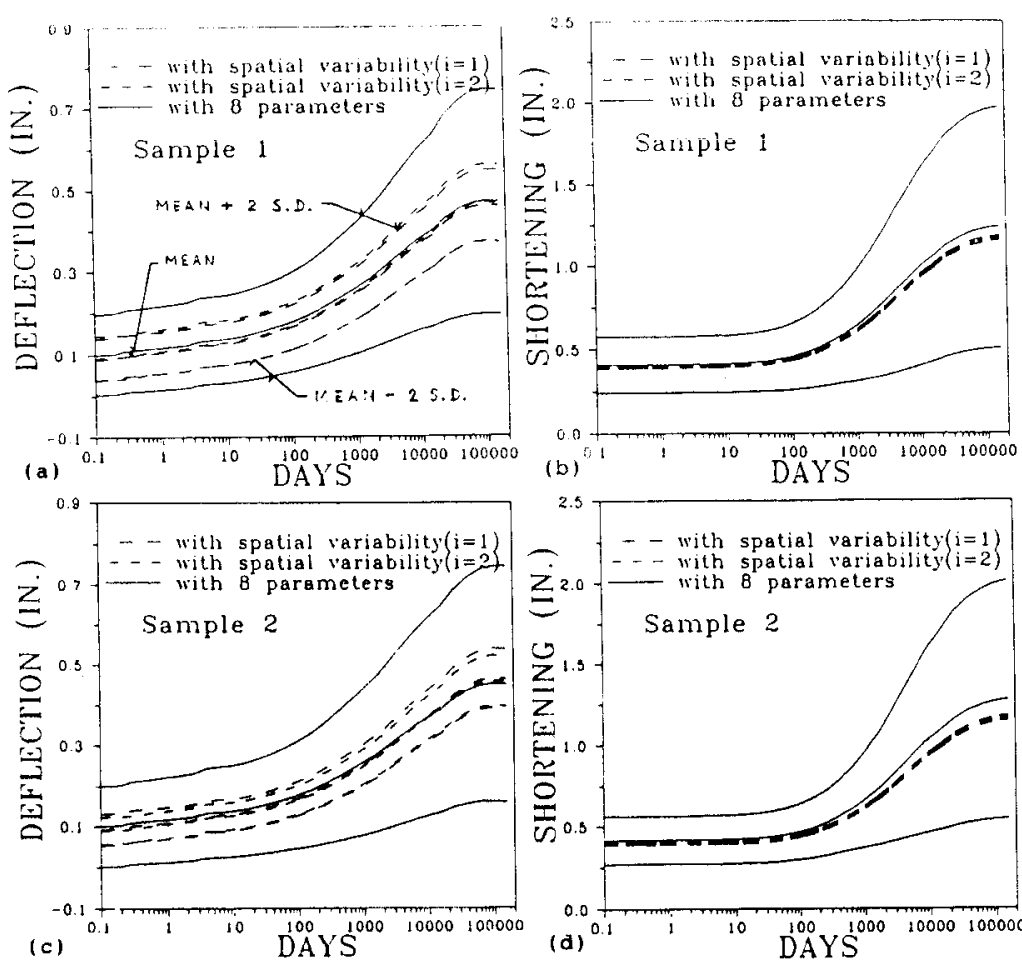

FIG. 1. Means and Confldence LImits of Deflectlon History Considering elther Random Varlability of Elght Materlal Parameters or Spatial Random Varlabillty

sen as $L_{i x}=140$ in. $(6.10 \mathrm{~m})$ and $L_{i y}=750 \mathrm{in}$. $(19.0 \mathrm{~m})$ (these roughly correspond to the depth of the bridge and the length of the half span of the bridge).

Figs. 1 and 2 show the results of calculations obtained under the assumption that all the data and random characteristics other than those of $\alpha$ are exactly the same as in the preceding paper (Bažant and Kim 1989). The parts show the predicted means and $95 \%$ confidence limits for deflections of segment number 17 and the axial shortening at midspan of the box girder as functions of time (in the logarithmic scale). All the figures show the results considering either one or two random components in (2). We see that the number of components has relatively little effect, which a posteriori justifies not using more components. In Figs. 1 and 2, the curves labeled "with 8 parameters" give the variability taken from the preceding paper, without any effect of spatial variability of $\alpha$. In Fig. 1, the curves labeled "with spatial variability" give the results for the case in which only $\alpha$ is a spatial random field and all the other material parameters have their mean values. The results labeled "sample 1 " and "sample 2 " are calculated for two different randomly generated latin hypercube samples of the material parameters or two different randomly generated latin hypercube samples of spatial random field as parameters entering $\alpha$. In Fig. 2, the curves labeled "plus 

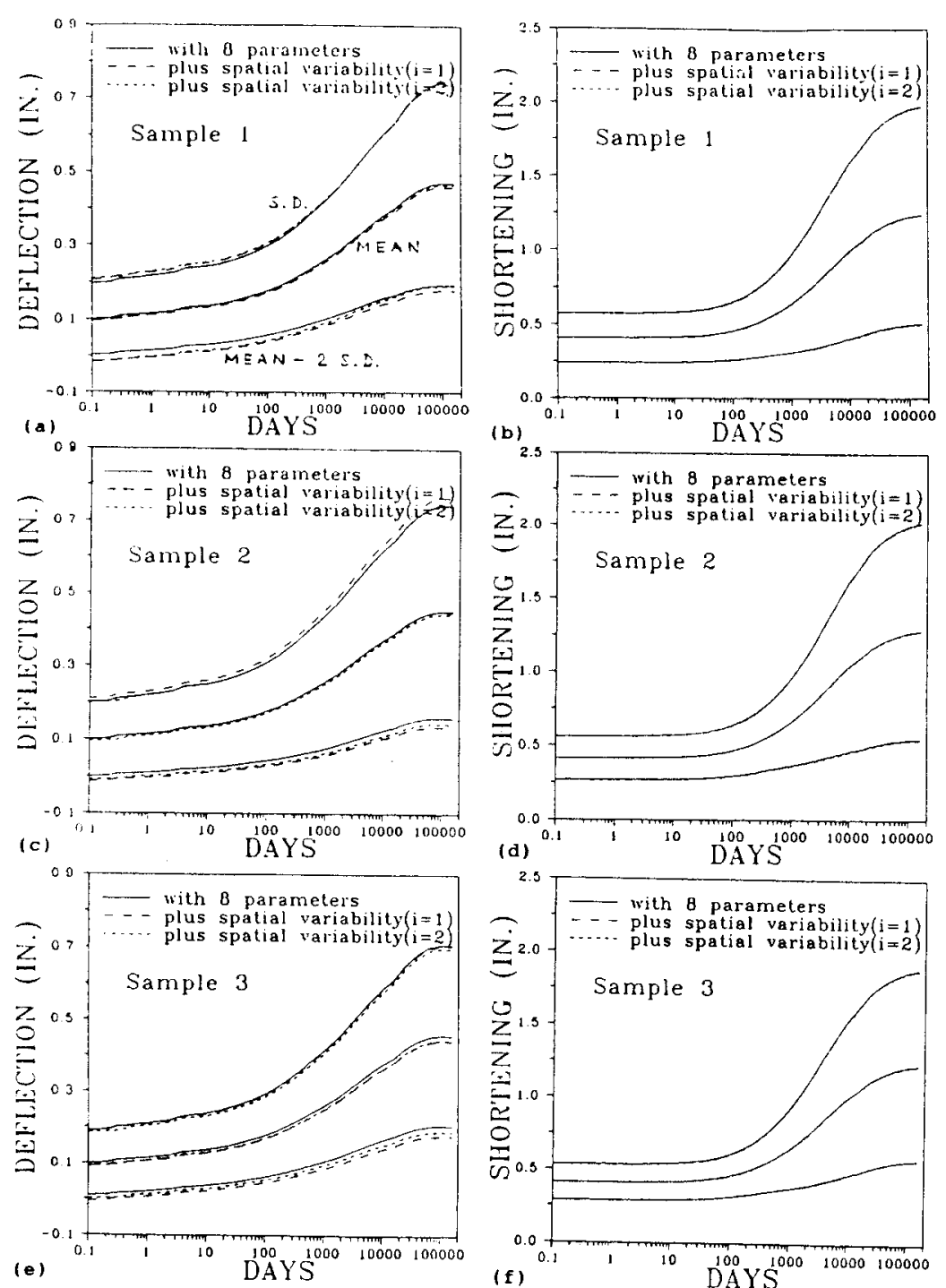

FIG. 2. Predicted Means and Contidence Limits Considering Random Spatia Variability Together with Random Varlability of Elght Parameters Compared to Result for Eight Parameters as Only Random Parameters

spatial variability" are the results obtained when both the eight random material parameters and the additional parameters defining the random field $\alpha$ are taken into account as simultaneous random variables. The results labeled "sample 1," "sample 2," and "sample 3" are calculated for three different samples of the material parameters and three samples of the spatial random field. Although an infinite number of random samples would be needed to get the exact statistical characteristics of the response, three samples probably suffice for practical prediction of bridge response.

\section{Conclusions}

Even though our estimate of the statistical parameters of the random field cannot be substantiated by any available measurements, one can conclude from Figs. $I$ and 2 that the effect of spatial variability taken alone is considerably smaller than the effect of random variability of material parameters considered in the preceding paper (Fig. 1).

Furthermore, from Fig. 2 one can see that the effect of adding the spatial variability to the effect of the random material parameters is only minor, and negligible from the practical viewpoint.

As an overall conclusion, the effect of random spatial variability of the compliance magnitude is rather small compared to the effect of the random statistical variability of the material parameters considered in previous work. In practice it can be neglected, although calculation of the effect of the random spatial variability by the latin hypercube sampling method is not difficult and can be easily included in the analysis.

This conclusion also justifies considering the random spatial variability only in a very crude manner, e.g. not bothering to take into account many spectral components of the power response spectrum of the spatial autocorrelation function.

\section{ACKNOWLEDGMENT}

Partial financial support under U.S. National Science Foundation Grant No. MSM-8815166 to Northwestern University is gratefully acknowledged. The calculations were supported from the Center for Advanced Cement-Based Materials at Northwestern University.

\section{Appendix. References}

Bažant, Z. P., and Kim, J.-K. (1989). "Segmental box girder: Deflection probability and Bayesian updating." J. Struct. Engrg., ASCE, 115(10), 2528-2547.

Shiu K N Taniel J.P and Russell, H. G (1983) "Time-dependent behavior of segmental cantilever concrete bridges." Report, Construction Technology Laboratories, Portland Cement Association, Skokie, III. 